\title{
A GEOMETRIC THEOREM AND ITS APPLICATION TO BIORTHOGONAL SYSTEMS
}

\author{
ANGUS E. TAYLOR
}

1. The geometric theorem. Let $E_{n}$ denote an $n$-dimensional Euclidean space. A set of points in $E_{n}$ may constitute an $E_{n-1}$; if so, it will be called a plane in $E_{n}$. We shall sometimes denote planes by $p_{1}, p_{2}, \cdots$ Also, if $x_{1}, \cdots, x_{n-1}$ are linearly independent vectors emanating from a common point $O$ in $E_{n}$, the plane through $O$ and containing these vectors will be denoted by $E\left(x_{1}, \cdots, x_{n-1}\right)$. A plane divides $E_{n}$ into two closed half-spaces each having the plane as boundary, but otherwise having no points in common. If $S$ is a point set in $E_{n}$, a plane $p$ is called a supporting plane of $S$ if (1) $S$ lies in one of the closed half-spaces determined by $p$, and (2) the distance between $S$ and $p$ is zero.

ThEOREM 1. Let $S$ be a bounded and closed point set in $E_{n}$. Let $O$ be a point of $E_{n}$, and suppose that the set consisting of $O$ and $S$ does not lie in any plane. Then there exist $n$ linearly independent vectors $x_{1}, \cdots, x_{n}$ emanating from $O$, with terminal points $P_{1}, \cdots, P_{n}$ in $S$, and $n$ planes $p_{1}, \cdots, p_{n}$ satisfying the following conditions: For each $i$ (a) $p_{i}$ contains $P_{i}$; (b) $p_{i}$ is parallel to $E\left(x_{1}, \cdots, x_{i-1}, x_{i+1}, \cdots, x_{n}\right)$; (c) $p_{i}$ is a supporting plane of the set consisting of $O$ and $S$.

PRoof. Consider any $n$ points $P_{1}, \cdots, P_{n}$ in $S$. Let $x_{i}$ be the vector from $O$ to $P_{i}$. Form the parallelepiped determined by the vectors $x_{1}, \cdots, x_{n}$. As a figure in $E_{n}$ this parallelepiped has a certain content, which is a function of $P_{1}, \cdots, P_{n}$, say $V\left(P_{1}, \cdots, P_{n}\right)$. Because of the hypothesis concerning $O$ and $S$, there is at least one choice of $P_{1}, \ldots, P_{n}$ for which the parallelepiped is nondegenerate; $V$ therefore assumes a positive value. Now $V$ is a continuous function of the variables $P_{1}, \cdots, P_{n}$, each of which ranges over the compact set $S$. It follows that $V$ assumes a positive absolute maximum value. Throughout the remainder of the proof we shall use $P_{1}, \cdots, P_{n}$ to denote a set of points in $S$ at which $V$ attains its absolute maximum. The fact that the maximum is positive then implies that the vectors $x_{1}, \cdots, x_{n}$ are linearly independent.

Let $p_{i}$ be the plane through $P_{i}$ parallel to $E\left(x_{1}, \cdots, x_{i-1}\right.$, $\left.x_{i+1}, \cdots, x_{n}\right)$. The parallelepiped of maximum content lies between these planes, each of a pair of opposite faces lying in one of the planes.

Presented to the Society, November 30,1946; received by the editors November $12,1946$. 
We have to prove that $p_{i}$ is a supporting plane of the set consisting of $O$ and $S$, that is (since $O$ is not on $p_{i}$ ), that $O$ and any point of $S$ both lie in the same one of the two closed half-spaces determined by $p_{i}$. Suppose that for some point $Q$ of $S$ this were not the case. Consider the parallelepiped determined by the vectors $x_{1}, \cdots, x_{i-1}$, $O Q, x_{i+1}, \cdots, x_{n}$. Its content would evidently be greater than that of the parallelepiped determined by $x_{1}, \cdots, x_{n}$, in contradiction to the maximal property of the latter. For, the $n$-dimensional content of a parallelepiped is equal to the $(n-1)$-dimensional content of one of its faces multiplied by the distance between the plane of this face and that of the face opposite. The two parallelepipeds which we are comparing have a face through $O$ in common, but the distance from $O$ to $p_{i}$ is less than the distance from $O$ to the parallel plane through $Q$. The proof of Theorem 1 is now complete. ${ }^{1}$

2. Normed linear spaces. Let $X$ denote a real normed linear space. ${ }^{2}$ In this section we shall prepare the way for the proof of the theorem in the next section by discussing certain matters pertaining to $n$-dimensional linear subspaces of $X$. Let $Y_{n}$ denote such a subspace, and let $y_{1}, \cdots, y_{n}$ be $n$ linearly independent elements of $Y_{n}$, so that any $y$ in $Y_{n}$ is uniquely representable in the form

$$
y=e_{1} y_{1}+\cdots+e_{n} y_{n} .
$$

If in an $n$-dimensional Euclidean space $E_{n}$ we introduce a rectangular coördinate system, we may set up a correspondence between the element $y$ of $Y_{n}$, as given by (2.1), and the point with coördinates $\left(e_{1}, \cdots, e_{n}\right)$ in $E_{n}$. This correspondence is one-to-one and bicontinuous. We state the following lemmas, leaving the proof of the first one to the reader.

LEMMA 2.1. The points of $E_{n}$ which correspond to the elements $y$ of $Y_{n}$ for which $\|y\| \leqq 1$ form a bounded and closed convex set $S$ which is symmetric about the origin. If $\|y\|<1$, the corresponding point of $E_{n}$ is an interior point of $S$.

Lemma 2.2. Let $S$ be the set referred to in Lemma 2.1. Let $P_{0}$ be a point on the boundary of $S$, and $p_{0}$ a plane of support of $S$ through $P_{\theta}$. Denote by $p$ the plane parallel to $p_{0}$ and through the origin of $E_{n}$. Let $y_{0}$ be the element of $Y_{n}$ corresponding to $P_{0}$, and denote by $M$ the set of

1 The referee brought to my attention the fact that a theorem similar to Theorem 1 , for the special case in which $S$ is a convex body with the point $O$ as a center of symmetry, was announced by M. M. Day, in Bull. Amer. Math. Soc. Abstract 51-11-237.

2 S. Banach, Théorie des opérations linéaires, Warsaw, 1932, p. 53. 
elements of $Y_{n}$ corresponding to points of $p$. Then there exists a linear functional $f_{0}$ defined on $X$ with the properties (1) $\left\|f_{0}\right\|=1$, (2) $f_{0}\left(y_{0}\right)=1$, (3) $f_{0}(y)=0$ for each $y$ in $M$.

Proof. Since $p_{0}$ does not pass through the origin, it may be described by an equation of the form $a_{1} e_{1}+\cdots+a_{n} e_{n}=1$. The points of $p$ will then satisfy the equation $a_{1} e_{1}+\cdots+a_{n} e_{n}=0$. By means of the correspondence (2.1) we define a linear functional $\phi_{0}$ on $Y_{n}$ by the equation $\phi_{0}(y)=a_{1} e_{1}+\cdots+a_{n} e_{n}$. It is easily proved, by using the last sentence of Lemma 2.1 and the fact that $p_{0}$ is a supporting plane of $S$, that $\left|\phi_{0}(y)\right| \leqq 1$ if $\|y\| \leqq 1$. Note that $\phi_{0}\left(y_{0}\right)=1$. Consequently, by the Hahn-Banach theorem, ${ }^{3}$ there exists a linear functional $f_{0}$ defined on $X$, having $\left\|f_{0}\right\|=1$, and coinciding with $\phi_{0}$ on $Y_{n}$. This functional evidently meets the requirements of Lemma 2.2.

3. Biorthogonal systems. Consider a real normed linear space $X$, and the conjugate space $X^{*}$ of linear functionals defined on $X$. A pair of ordered sets $\left\{x_{1}, x_{2}, \cdots, x_{n}\right\} \subset X$ and $\left\{f_{1}, \cdots, f_{n}\right\} \subset X^{*}$ is called a biorthogonal system if $f_{i}\left(x_{j}\right)=1$ when $i=j$ and $f_{i}\left(x_{j}\right)=0$ when $i \neq j$ $(i, j=1,2, \cdots, n)$. If in addition $\left\|x_{i}\right\|=\left\|f_{i}\right\|=1(i=1,2, \cdots, n)$, we shall call the pair of sets a biorthonormal system.

THEOREM 2. Let $Y_{n}$ be an n-dimensional linear subspace of $X$. Then there exists a biorthonormal system $\left\{x_{1}, \cdots, x_{n}\right\},\left\{f_{1}, \cdots, f_{n}\right\}$ with $x_{1}, \cdots, x_{n}$ in $Y_{n}$.

Proof. Introduce the mapping of $Y_{n}$ on $E_{n}$, and the set $S \subset E_{n}$ as defined in \$2, Lemma 2.1. With $O$ the origin in $E_{n}$, let $P_{1}, \ldots, P_{n}$ and $p_{1}, \cdots, p_{n}$ be points and planes related to $S$ as described in $\$ 1$, Theorem 1 (here $O$ and $S$ do not lie in any plane). Let $x_{1}, \cdots, x_{n}$ be the elements of $Y_{n}$ corresponding to $P_{1}, \cdots, P_{n}$. The points $P_{j}$, $j \neq i$, are in the plane through $O$ parallel to $p_{i}$. By Lemma 2.2 it follows that there exists a linear functional $f_{i}$ such that $\left\|f_{i}\right\|=1, f_{i}\left(x_{i}\right)=1$, $f_{i}\left(x_{j}\right)=0, j \neq i$. This completes the proof of the theorem.

University of California at Los Angeles

\footnotetext{
8 Banach, loc. cit. Théorème 2, p. 55.
} 